\title{
Entrepreneurship Education Platform Design: The Relationships between Autodidactic Affordances and Experiential Learning Needs
}

\author{
Francezca Dagoc \\ DiLab, San Diego State University \\ fdagoc@sdsu.edu \\ Vanessa Roy \\ DiLab, San Diego State University \\ vroy6916@sdsu.edu
}

\author{
Kaveh Abhari \\ DiLab, San Diego State University \\ kabhari@sdsu.edu \\ Elijah Nobis \\ DiLab, San Diego State University \\ enobis0387@sdsu.edu
}

\begin{abstract}
Along with recent technological innovation in education, Entrepreneurship Education Platforms (EEPs) have emerged as an effective solution to prepare students for entrepreneurial success. However, due to novelty, less is known of the success of EEPs regarding user experience, especially when these platforms offer self-regulated learning environments. In response, this study is an attempt to offer a framework to guide user experience design and evaluation of EEP. This study discusses the relationships between autodidactic affordances (administration, acquisition, application, and assessment affordances) and experiential needs (sensory, temporal, interactional, cognitive, behavioral, emotional, and metacognitive experience) and their importance in the success of EEP. The findings offer nuance to assessing the effectiveness of EEP and other online self-regulated learning environments.
\end{abstract}

\section{Introduction}

Entrepreneurship has played a pivotal role in economic development and innovation in business. As a result, several institutions have stressed the importance of teaching entrepreneurship to students as early as possible through entrepreneurship education [1], [2]. Latest trends of online education, including those due to COVID-19 pandemic restrictions, have shifted entrepreneurship education to digital environments called entrepreneurship education platforms or EEPs. Although recent studies highlighted the importance of these platforms, limited research investigates user experience on EEPs. EEPs represent a rich case such that current literature falls short in its theorization. The present study fills this gap by examining user experience on the EEP BizzyB.
More specifically, this study presents a user experience design framework that assesses the relationship between four autodidactic affordances provided by the platform (administration, acquisition, application, and assessment affordances) and six experiential learning needs of end-users (sensory, temporal, interactional/social, cognitive, behavioral, emotional, and metacognitive experiences). This framework is contextualized to entrepreneurship education goals and self-regulated learning (SRL) capabilities through a case study of one EEP. Both its users and developers were interviewed in order to create a framework guiding future research and development in this area. Our framework theorizes the relationships between affordances and experiential needs sought by users. The findings suggest that accommodating these needs through certain affordances is vital to offering satisfying user experience on EEPs and similar learning platforms. This research contributes to the platform design theories as well as entrepreneurship education literature by offering a user-based framework to study EEP and similar learning platforms. Moreover, the proposed framework can be used to improve the design of other educational models and inform the related UI/UX practices. More generally, our research proposes a new method to study, model, and evaluate digital platforms through a user experience lens and offers a new interpretation of the Needs-AffordancesFeatures perspective.

\section{Background}

Information systems literature calls for research on digital platforms design in different domains [3], [4]. Education, and entrepreneurship education (EE) in particular, is one of those domains [5]. EE is the process of promoting insight, knowledge, confidence, and skills to encourage individuals to act on and acknowledge commercial opportunities [6], [7]. EE 
seeks to facilitate the development of an entrepreneurial mindset and competence [8] through a range of teaching methods and strategies [5], including design thinking techniques that emphasize collaboration [9] and project-based learning [10]. EE models vary depending on the type of learner being taught and the intended learning outcomes [11]. However, these models use the power of SRL and digital technology to encourage creative problemsolving and minimize the fear of failure to build an entrepreneurial mindset [10], [12]-[14]. To that end, prior studies have discussed the prominence of this approach and resulting success of EE programs. Research has shown that this approach positively influences student attitudes toward entrepreneurial intention [15], and thus affects their career paths, with many becoming self-employed, joining startups, or owning their own businesses after college [16]. However, as with other educational programs, further research is needed to systematically implement this approach in digital settings.

\subsection{EE in Digital Settings}

With the recent advancements in technology and limitations imposed by the COVID-19 pandemic, education has shifted to digital learning environments. Following this trend, EE has also begun utilizing online learning to connect with a variety of students on a digital level. Research has shown that EE in digital environments has significant advantages over EE in more traditional settings [8], [9], [10]. One such study found that the usage of mobile classroom response system technology within EE was proven to be effective in increasing entrepreneurial intention, capability, performance, and involvement in students [8]. Similarly, another study showed the effectiveness of an online entrepreneurship program in developing entrepreneurial knowledge and mindset [1]. Nevertheless, there is still limited research available that evaluates EE in digital settings, especially in SRL environments [1], [17].

\subsection{Self-Regulated Learning on EEP}

Recent scholarship has focused on investigating behavioral components of entrepreneurship [11]. Such behavioral components have been used as a form of evaluative measure for how effective EE programs supplement entrepreneurial competencies in learners. One major behavioral component of emerging EEPs is self-regulated learning (SRL).

SRL refers to the process by which learners are allowed, empowered, and encouraged to plan and manage the learning process individually. This effective component involves four phases: planning, preparation, control, and reflection. Each phase requires cognitive, metacognitive, motivational, behavioral, and contextual actions [18]. SRL has been shown to be effective in educational programs [9], [19], [20], positively influencing student performance (e.g., improved learning outcomes [21]).

The positive impact of SRL is not limited to academic achievements. Previous research has indicated a positive relationship between selfregulation and entrepreneurship. Self-regulatory characteristics were prominent in entrepreneurs who were found to have more moral awareness [22]. Entrepreneurs who develop self-regulatory characteristics were shown to effectively work in teams and participate in collective decision-making [23]. Relevant to the study at hand, previous research has analyzed SRL and its impact on EE. SRL has been shown to have a positive effect on entrepreneurial learning outcomes [9], with elements in SRL such as self-efficacy positively influencing students' entrepreneurial attitudes [24] and intentions [25]. Because SRL can assist students in accomplishing learning goals, many developers incorporate SRL in the design and implementation of EEPs [9], [26]. This inclusion warrants further research and investigation related to the affordances provided by EEPs, thus informing the present study's focus on an EEP emphasizing SRL.

\subsection{The importance of UX to support EEP}

User experience (UX) is conceptualized as the interactions an end-user may have with a certain product or service [27]. UX goes beyond usability, taking into consideration the needs of users. Specifically, UX takes into account the emotional, subjective, and temporal components users have in their interactions [28], making UX particularly helpful in guiding the interface design of a product. In recent years, many researchers have used UX concepts to assess or improve products or interface designs [27]. For example, one study analyzed user experience through a four-dimensional evaluation model that examined usability, gaming experience, learning experience, and adaptivity in a digital education game (80Days) [29]. These UX concepts have been utilized in various settings, including self-regulated, digitally enabled educational settings [30].

Past research has also evaluated UX in a digital learning environment. For example, one study evaluated UX in a collaborative virtual environment to understand and enhance collaborative learning [31]. The study compared college students' user experience and aligned it with their performance outcomes in a virtual world (Second Life), a learning platform 
(Blackboard), and face-to-face learning. The results showed that, although user experience in Second Life was positive, the usability of the virtual world was less successful for students compared to Blackboard.

This array of past research shows clear relationships between user experience and platform affordances related to SRL. Affordances are known as action possibilities that a user perceives in interaction with a particular object within the platform [32]. In the context of UX, interface affordances are useful in evaluating the success of an interface platform's end goals and help users visualize potential actions [33], [34]. From an SRL standpoint, affordances give opportunities for self-directed, self-regulated, and/or self-managed learning activities that can be either enabled or facilitated by the platform features [35].

Previous research has demonstrated the importance of studying affordances in educational settings - for example, to improve interactivity between users [36] and develop more communicative fluency and activity [6]. Other studies have investigated affordances as a way to further understand users of a particular application or service [31], [37]. When thoroughly examined, affordances also allow researchers and developers to better understand the views and experiences of educators in learning environments [37]. In addition, past research has shown the significance of evaluating UX from an affordance perspective in virtual educational settings, especially on SRL platforms [31], [29], [36], [37]. However, limited research has applied these investigations to EEPs. Addressing this gap and the increasing popularity of EEPs, the present study examined UX of EEPs from an affordance perspective. Therefore, this study addresses the following research questions: (a) What are the key affordances expected by users on EEP? (b) How do these affordances satisfy experiential learning needs?

\section{Methods}

An exploratory case study was performed to answer the above research questions. A popular EEP, BizzyB, was closely examined and data were collected through observation, email interviews with users, and one-toone interviews with developers. The study includes interviews with three developers who have led the design, development, deployment, and maintenance of the platform since its launch in 2015. 120 college students were also invited to use the platform as new users to participate in this study. To minimize selection bias and the effect of experience, only new users were selected, and they were given the same amount of time (two months) to use the platform. New $B i z z y B$ users received open-ended questions on their perception of $B i z z y B$ as a whole, their view of the platform design, their usage experience, and their comparison of the platform with other educational platforms. These questions included, for example, "What was your overall impression of BizzyB?", "How do you describe your experience with this platform?", and "How would you change the design of this platform?". Additionally, developers received open-ended questions on the evolution of the platform's design along with questions about the feedback they received from experienced users and educators. Questions that were given to developers ranged from, "Can you tell me about any interesting feedback or stories you received from end-users?" to follow-up questions such as "What specific features did you design that the users enjoy most and why?".

The final dataset contained 44 email interview responses from end-users and 3 interview transcripts with developers. This large sample was necessary to capture a wide range of opinions because the email interview did not allow follow-up, probing questions. A three-step content analysis approach was used that included open-coding, axial-coding, and selective coding. This approach is the most applicable given the lack of existing frameworks that have already identified affordances and needs in EEP context. Also, previous work on similar platforms has utilized a similar data analysis strategy [31],[37]. We used an open-coding method to extract the key user experiences, affordances, and needs associated with each user story. Afterward, the axial coding allowed the discovery of hierarchies among the codes, which were then classified into key categories. Relationships between the key categories of affordances and experiences were then identified. Finally, these findings informed the creation of a user experience design framework for EEPs.

\section{Case Study}

This framework was developed using an investigation of a popular EEP, BizzyB. This platform contains an extensive array of features and learning paths for different age groups, providing students from middle school to college with a collaborative environment to learn, develop, and exercise entrepreneurial skills. This platform consisted of a diverse set of features and tools to aid users in completing an entrepreneurial project, either independently or with minimum instruction from a teacher. To help users with projects, $B i z z y B$ offers mastery lessons along with quizzes, exercise, sample projects, reflections, and mentorship opportunities.

A major component of BizzyB is its project journal. BizzyB's FabFive project journal encourages users to utilize a design-thinking process that emphasizes a story-based creative-cycle approach. 
The project journal template entails five entrepreneurial conceptual activities that mirror company executive suites: Idea (CEO), Product (CTO), Marketing (CMO), Sales (CSO), and Goals (CFO). Users can practice leadership skills through completing and facilitating tasks within every phase. Each learning project on BizzyB uses these phases, along with five predefined activities under each phase. Activities ask users to brainstorm and research ideas and then use that research to create and upload descriptive content, images, or videos. The learning activities are self-regulated and fulfill the requirements of a successful shortened business model by the end of each project. By completing each project, users can create a presentation pitch as their final outcome. Completed projects can be entered in online showcases and contests.

Each learning activity is supported by learning options such as Instructions, Tips \& Examples, a Comment Sidebar, Virtual Mentoring, Feedback Surveys, and Idea Credits. The platform also collects users' profile data, soft skills, innovation competencies, usage data (interactive events), and feedback data (progress) to offer more personalized learning experiences. Volunteer mentors offer recommendations to users on how to reach a reasonable level of proficiency in each phase. The platform allows mentors to communicate with users virtually via the project journal (and sidebar commenting). Mentors can also recommend learning content to the users from the BizzyB library. Additional features like self-assessment tools allow users to track their progress, assess their soft skills, and build a digital portfolio with reports that showcase their accomplishments. The extensive number of features introduced above that emphasize SRL made BizzyB the ideal platform for this study.

\subsection{BizzyB Design Background}

Prior to examining BizzyB's features in-depth, it was essential to gain an overall understanding of the platform's design and background. As expected, BizzyB developers went through numerous UI/UX sketches, specifications, and prototyping before releasing its first version. After investigations of procedural and technical difficulties encountered by users within the system, various interface design modifications were implemented. Further design refinements were identified and implemented based on benchmarking best practices and feedback solicited from entrepreneurs, mentors, and researchers. Specific improvements included the creation of new functionalities such as live/real-time tools like live chats as well as collaboration mechanisms to facilitate user engagement and enhance collaboration. Options for participation in contests and hackathons were also added after the first build. Other changes involved simplification of some features and processes within the system, such as reducing the number of complex tools, simplifying the sign-up process, and optimizing the number of activities for each project. As with many platforms, BizzyB was still considered a work in progress by its developers at the time of the interviews.

Despite the numerous improvements, developers indicated that they faced several design challenges. One significant challenge discussed was a complication in creating a user-friendly and connected application while still retaining an assortment of features and learning tools. Many experienced users faced a learning curve and had a "challenge to express what they've learned" as BizzyB, "seemed very different from traditional learning." This presented a problem for developers, as they needed to design platform features to effectively support the SLR process. Developers also had difficulty adhering to navigational needs of the four different groups of users. Developer A remarked that, "Some could adapt to it very quickly" while others "could not adapt to it quickly but could do it eventually." Also, design for the onboarding process presented user engagement issues as "the flow wasn't very intriguing" when students were invited by teachers or groups. Developer B commented on the complexities of nesting groups within learning organizations while maintaining synchronized data in the system. More importantly, prioritization of different users' needs presented tradeoffs for developers. Prioritizing the needs of users led to less design support for navigational issues encountered by educators. In fact, students had a greater aptitude for navigating the application and digital learning platforms in general. Likewise, as the platform became more customizable for users, developers had difficulty adhering to specific customization requests made by school administrators. While most challenges developers have encountered have been remedied, design problems associated with usability and user satisfaction remain in need of revision. This case study addresses this gap by gaining further insight into user needs and providing specific user experience recommendations that can guide future interface redesigns for BizzyB.

\subsection{Features and Affordances Examination}

We closely examined the platform to identify affordances and constraints along with their associated features. The identified affordances, constraints, and features were developed using an SRL affordance framework and the Needs-Features-Affordances (NAF) perspective [38]. The NAF framework argues that a platform designer should start with users' goals 
to then work backwards to plan a set of affordances and their features to meet the needs of users. At the same time, those needs can motivate the use (or actualization) of certain affordances. The higher the rate of actualization, the more successful the design. Therefore, both the design and evaluation of a digital artifice, an EEP in our case, should start with the careful identification and examination of the interactions between the platform's affordances and needs of its users. Following this assumption, the features and the affordances enabled by the platform were first identified, followed by the features supporting affordances mainly related to SRL. Table 1 summarizes the key identified affordances, referred to in this study as autodidactic affordances.

The autodidactic affordances identified in this study enabling or facilitating SRL were labeled as administration, acquisition, application, and assessment affordances. For administration affordances, users identified their need for features that help them in planning their learning journey and developing a sense of control over their learning experiences. The analysis showed that examples of features enabling or supporting administration affordances include project customization, profile management, group administration, and planning options such as a calendar and reminders.

Acquisition affordances were shown to be associated with components that assist users with knowledge and content acquisition. User responses revealed that (knowledge) acquisition affordances could be egocentric or allocentric. Egocentric affordances were associated with those features enabling self-paced learning such as searching, browsing, and reading educational content in BizzyB's library. Allocentric affordances facilitated group learning, for example, through sourcing information, brainstorming, and seeking feedback.

Features enabling users to put their knowledge into practice are related to the platform's application affordances. These features promote hands-on activities that require ideation, collaboration, and communication with teammates. Activities enabled by these features include posting and revising ideas, attaching video presentations, and participating in discussions. The application affordances are the affordances that differentiate BizzyB and similar EEPs from typical learning platforms. These affordances offer experiential learning opportunities through which users gauge their understanding in a simulated environment rather than going through arbitrary knowledge assessment. This was also noted by users as one of the critical components of this platform.

Lastly, assessment affordances were related to feedback features that allowed users to assess their knowledge and track their personal and group progress. These affordances are enabled by features like lesson exercises, quizzes, assignments, selfassessment surveys, a progress dashboard, a mentorship feedback board, and contests for users to present their work.

Table 1. Affordances

\begin{tabular}{ll}
\hline AFFORDANCES & ACTIONS \\
\hline Administration & $\begin{array}{l}\text { Users join, create profile, connect to others, } \\
\text { build team, customize some services. }\end{array}$ \\
\hline Acquisition & $\begin{array}{l}\text { Users access content, read content, discuss } \\
\text { content, share content, reflect on content. }\end{array}$ \\
\hline Application & $\begin{array}{l}\text { Users apply or create knowledge within } \\
\text { projects - individually or collaboratively- } \\
\text { based on what have learned }\end{array}$ \\
\hline Assessment & $\begin{array}{l}\text { Users participate in assessments, track } \\
\text { progress, peer review, reflect, receive } \\
\text { feedback from mentors, awarded badges. }\end{array}$ \\
\hline
\end{tabular}

The affordances identified in Table 1 provide a foundation to examine how BizzyB fulfills users' needs and how users' needs motivate the use of the features that provide said affordances. Building on this, the next section explores the relationship between experiential learning needs and autodidactic affordances to identify the experiential learning values of EEP in SRL environments.

\subsection{Experiential Values of Affordances}

After identifying the above affordances, we connected each affordance group to its experiential values. This step was essential to build an understanding of the relationship between users' needs and the affordances provided by $B i z z y B$ as experiential learning values motivate the initial and continued use of a platform (cf. [38]). The axial coding of the interview data helped us to identify seven experiential values for autodidactic affordances: sensation, time, connection, cognition, action, emotion, and metacognition. We should note that the goal was not to directly evaluate $B i z z y B$ but to understand its users' needs and expected values in using EEPs. This means that the results of the content analysis revealed that participants sought experiences related to, for example, connection and emotion from the previously identified affordances - regardless of user's awareness of these values or actualization of these affordances on the BizzyB platform.

Throughout the interview, users shared the most about sensorial values (i.e., the platform look and feel) related to administration affordances. For example, most users discussed their interface design preferences and the need for more (or less) interactive navigation features. The comments ranged from reactions to the user interface components and style to visual cues guiding the activities. User 28 argued, "Users should not be fighting to understand the system when they could be learning the material and utilizing the 
features. However, the [interface] is pleasant to use, once the user understands how the site works." Other comments related to the need for customization - to meet users' UI preferences - and the need for visual referential elements such as video, audio, or image instructions and examples. Sensory values were also highlighted in discussions of administration features from profile appearance to work presentation. For example, users stressed the importance of intuitive buttons/icon design and the need for creating better UI for team management.

The participants discussed the importance of temporal design elements such as progress monitoring through a progress bar, reminders, and a timer. These comments were classified into the temporal value of design - which is an understudied topic in affordances literature. In the context of application affordances, participants discussed how time management, intuitive design, and simplified processes could create value for users. This was expected as application affordances were mainly associated with time sensitive activities, especially among users participating in a contest or completing activities along with classmates in a program.

When discussing the social value of BizzyB, users emphasized features allowing collaboration between learners or between learners and mentors. Examples of these features included contests, chats, comments, and direct communication with mentors. For instance, User 33 explained that they found the mentor feature helpful as users, "...can gain leadership experience" and "have someone with experience to give them advice or answer their questions." Users primarily discussed these features in the context of acquisition and application affordances. These features were shown to support users in networking and collaboration with both peers and mentors during the knowledge acquisition process.

Users connected perceived cognitive value in BizzyB's knowledge acquisition, application, and assessment affordances. Cognitive values were twofold: the participants noted the importance of the platform affordances in learning content pertaining to both entrepreneurship and the project structure. The practicality of this feature provides users with the ability to put their knowledge into practice systematically. Users discussed how the platform satisfied their need for guidance and offered various tools like the online library to help them with knowledge acquisition and retention. User 10 remarked that they liked how lessons, "walk you through each step" and lesson guides "show you how to fill out $\mathrm{x}, \mathrm{y}$ and z." Users also identified ways that the cognitive value of $B i z z y B$ could be improved with variations in self-assessments, increased learning opportunities from mentors, better descriptions for project templates, and easier to understand instructions for how to use the dashboard (e.g., a soft skills report).

The core value of the BizzyB experience rests on its capability to offer a testbed for ideation and collaboration beyond traditional learning. This is closely related to the experiential and project-based learning features of the platform. The participants, for example, touched upon favoring goal-oriented design features associated with application affordances as that helped them test and apply their knowledge and skills. Users preferred affordances that related to features that assigned activities, broke them down into multiple tasks, and showcased user work and progress (e.g., contests and portfolios). Actions like providing or soliciting feedback, sharing work, and reporting the final project were among the top noted values. User 36 explained that these action-oriented features helped "...to make sure the users stay on the right track." These values are strongly associated with application and assessment affordances.

For emotional values, users said they liked elements that considered the feelings and psychological needs of users. This included the usage of inspirational quotes and completion badges available to the users. Participants noted feelings of control, belongingness, competence, achievement, enjoyment, excitement, and novelty. Many participants discussed emotional values in tandem with other values. For example, the emotional values of self-efficacy are closely related to social and behavioral values actualized after connecting to likeminded peers, learning by doing, completing a project, being encouraged by a mentor, personalizing a learning experience, and being awarded a badge.

Lastly, unique features related to the assessment affordances of BizzyB rendered metacognitive values due to their emphasis on self-evaluation and reflection. The assessment affordances that helped users become aware of their overall skills, strength, and behavior were mentioned among the top factors. User 14 referred to the user dashboard and preferred it over other learning platforms, because it provides "...a useful way of keeping track of what areas were worked on and what areas need improvement." Some examples of features supporting metacognitive experience formation include user dashboards and portfolios, as they offer the user an overview of their entrepreneurial archetype, competence, socioemotional and soft-skills, mentor feedback, and a progress log.

The findings also indicate interdependencies between the identified experiential values. For example, experiencing action-oriented values seemed dependent on the realization of cognitive, emotional, and metacognitive values along the way. In all three occurrences, application affordances motivated the 
realization of values related to $\mathrm{SRL}$, from being cognitively challenged and emotionally fulfilled to developing higher-order self-awareness and selfcontrol. This could be because SRL plays an influential role in the development of inspirational emotions, which in return results in metacognitive experiences. Additionally, the results revealed a commonality between all identified affordances due to the shared features enabling these affordances. This suggests the importance of an integrated approach to platform design and the evaluation of user experience according to the platform's affordances rather than its features. This also suggests the experiential value of a platform motivates its use, not its unique and novel features and functionalities.

\section{Discussion}

The above results informed the development of a framework guiding EEP user experience design and evaluation (Figure 1). This framework illustrates the relationships between SRL goals (plan, prepare, learn, and reflect) [18], EEP key features (account management, learning management, ideation and collaboration, and assessment and reflection), autodidactic affordances (administration, acquisition, application, and assessment), and experiential values (sensory, temporal, interactional, cognitive, behavioral, emotional, and metacognitive) needed in self-regulated EEP settings. The findings of the case study indicate that SRL goals motivate the use of EEP features, and, in turn, these features enable autodidactic affordances (cf. [32]). These affordances, if perceived, can be actualized by the users to fulfill their own experiential learning needs.

Moreover, data from new and experienced users revealed a gap between how users perceived the experiential values of $B i z z y B$ at different points in time. In benefiting from the platform affordances, it appeared that a new or novice user mainly perceives the sensorial, social, and emotional values of the platform (i.e. early experiential values). However, long-term users, after participating in and completing projects, may fully realize the experiential values of the platform in terms of its temporal, cognitive, behavioral, and metacognitive values (i.e., evolved experiential values). Interestingly, for these users, sensorial, social, and emotional values of the platform may be superseded by the evolved experiential values. This means users may be initially attracted to an EEP because of their own curiosity and/or the platform's user interface and sense of community. However, users may only continue to participate if they realize these evolved experiential values. Therefore, to maintain participation, EEPs must challenge its users cognitively and temporally and effectively engage them in hands-on activities and self-reflection. Based on this framework, the next section offers some recommendations pertaining to feature design and selection to enhance user experience on EEPs.

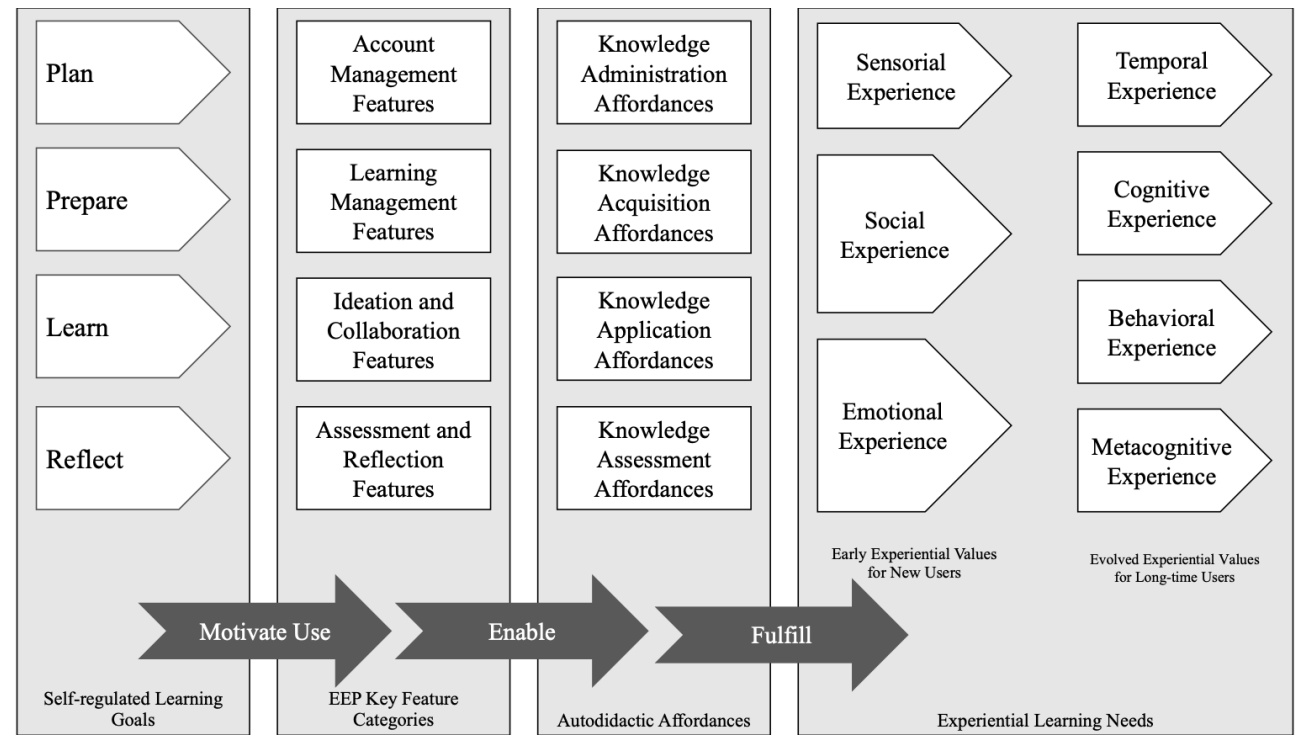

Figure 1. User experience design framework for EEP

\subsection{How to Enhance User Experience}

The results of the case study emphasize the importance of sensory experiences, especially when users first join and start using a platform. These experiences are enhanced by administration affordances as they allow users to create profiles, set goals, join groups, and start projects. Administration affordances can be enhanced 
by creating visual cues and simplifications of onboarding interfaces. New users may also benefit from an intuitive navigational design.

Temporal experiences could be enhanced through rethinking administration affordances of a platform. Features such as project-journaling, benchmarking temporal performance, and summarizing user progress visually could help users with timely actions to learn the value of time in entrepreneurship.

Social experiences could be improved by features that allow users to collaboratively interact with mentors or other users across the platform. These experiences are critical to the success of EEPs for two reasons. First, social experiences are key to encouraging new users to join and explore the platform. Experiences such as connecting and interacting with other users can motivate new users to utilize the platform more effectively. More so, social experiences can potentially lead to co-learning, peerto-peer mentorship, and project collaboration, actions that contribute to behavioral and metacognitive experiences. Therefore, including streamlines socialization and communication can help improve knowledge acquisition and application.

Cognitive experiences are also critical to the success of all learning platforms; EEPs are no exception. Cognitive experiences render the quality of learning outcomes and thereby need to be supported by a variety of affordances from acquisition to application. In the context of EEPs, features related to preparation, ideation, and collaboration play a vital role in enabling such affordances. The findings of the present case study suggest simplification, customization, and variation in ideation and collaboration tools are all important in enhancing cognitive experiences. However, it is important to acknowledge the tradeoff between offering more tools to support more experienced users and simplification to support those new to the platform.

Behavioral experiences form after users put their knowledge into practice in the form of projects. To enhance behavioral experiences, users need to have the tools that allow them to explore, create, experiment, and validate their entrepreneurial ideas beyond the typical learning process. Thus, the findings of this study suggest focusing on application affordances enabling successful project completion. Currently, application affordances on EEPs mainly offer ideation and collaboration opportunities. Therefore, EEPs and educational platforms alike can differentiate themselves by including features that allow users to define or select a framework for action (e.g., template for a business model). These features, beyond ideation and collaboration, could help users to set goals, personalize their learning, track their progress, validate their ideas, and present their work.
Hence, administration affordances can also help in the formation of positive behavioral experiences.

Emotional experiences could be enhanced by the features or content that reflect 'care' towards users, whenever appropriate. This case study found that many factors contribute to the formation of positive emotional experiences - for example, inspirational quotes, the design of mentorship pages, competency reports with goal setting, portfolios, and socializing. However, it would be naïve to assu31me these design features would trigger all users' emotions in the same way. For example, in this study, many features admired by some users were labeled as distractions or unnecessary by others. Furthermore, emotional experiences could be supported by two other factors: sense of control supported by administration affordances and self-efficacy supported by application affordances. Control and efficacy both encourage SRL behavior and are therefore important to support with features like personalization options, progress updates, achievement badges, and presentations.

Lastly, metacognitive experiences were found to play an influential role in maintaining user participation. Users indicated that application and assessment affordances encouraged them to develop self-awareness through formative and summative selfevaluation, respectively. Application affordances help users in aiming for a target without providing direct instruction on how to achieve their goal. The autonomy and flexibility of ideation and collaboration features helps users to practice planning, selfevaluation, patience, and persistence in achieving their goals. EEP designers can achieve this goal by reimagining self-evaluation affordances and related features such as progress visualization, personal skill logs, and project journaling. Highlighting users' accomplishments through encouraging elements such as badge completion could be also considered when increasing metacognitive awareness.

In sum, this framework promotes an 'experiencefirst' approach to the design and evaluation of educational platforms (cf. [39], [40]). Based on the findings of this study, user experiences should be designed first before the affordances and features are planned and created. This could potentially assist platform designers, particularly in educational settings, in instilling features that effectively motivate SRL behavior.

\subsection{Implications}

This research fills a gap in the literature by examining user experience on an increasingly popular platform type, EEP, and introduces autodidactic affordances to model affordances in SRL environments. This work presents a more thorough understanding of 
experiential values of using EEPs through exploring the relationships between these values and autodidactic affordances. We argue EEPs can be designed to plan, monitor, and evaluate SRL-driven experiences by accounting for the key relationships between experiential learning values and the various functional affordances. More specifically, administration affordances facilitate effective sensorial and temporal experiences. Social and cognitive experiences are mainly enhanced through acquisition and application affordances. Administration and application affordances are critical in planning positive behavioral and emotional experiences, and metacognitive experiences can be fostered by application and assessment affordances.

Our findings also suggest that platform developers should go beyond sensorial, emotional, and social experiences when designing and evaluating user experiences on collaborative learning platforms. This is a critical step in developing the next generation of educational platforms emphasizing open, projectbased, and self-managed learning environments. While this work emphasizes the importance of simple, intuitive, usable, and meaningful design as noted by previous studies [31], [29], it suggests that lasting values emerge from behavioral, temporal, and metacognitive experiences. However, these experiences may not fully develop unless the platforms succeed in enhancing sensorial, emotional, and social experiences first.

Our framework can inform future UI/UX research on emerging self-regulated learning educational platforms for two reasons: (a) SRL has been implemented in a similar manner on these platforms and (b) learners share similar experiential needs independent from the learning context. Our theorization of affordances and experiential learning needs also offers a new and systematic way to study learning platforms in the future. However, further development and validation of the framework in other contextual settings is necessary to ensure the transformability of the results.

Beyond theoretical implications, practitioners in both education and technology fields can adopt, test, and expand this framework to further investigate the role of EEPs in enhancing learning experiences and outcomes. Ideally, adoption of this framework would result in a more systematic understanding of EEP design, which in turn benefits alternative models of EE. Lastly, EEP developers can use this framework to analyze which experiential needs are the most important to address with the tools they are creating. That would help developers to identify the affordances that satisfy given needs and the features that then enable those affordances. Therefore, EEP developers should model how users would react to or benefit from different affordances before planning platform features. This has the potential to reduce the number of design iterations and maximize the chance of platform success and efficiency. Post-development, this framework may inform the evaluation and user test plans of EEPs.

\section{Limitations and Future Research}

While this study provides potential groundwork for future research, its limitations should be carefully considered. The results presented in this work were limited to a single case study and a limited number of interviews. Hence, using new cases to validate and extend this framework is necessary. This case was also based on a specific form of EE inspired by projectbased learning. Thus, other EEP with different educational models and learning strategies need to be included in future investigations. Likewise, the case study findings were drawn based on interview data from a homogenous group of users and a group of developers. Therefore, other data sources like tracking user behavior and surveying a representative sample of users would offer a more comprehensive explanation of user experiences. While this study is limited to learners, future studies should evaluate the experience of educators and mentors involved in EEPs as well. Additionally, this study only emphasizes learning affordances based on SRL activities presented within typical EEPs. Hence, future research should also investigate different forms of affordances. Most importantly, no causal relationship between identified concepts can be claimed based on this study. Therefore, conducting experiments to validate these relationships is another important research avenue.

Beyond an EEP context, future studies can use this framework to evaluate other learning platforms or virtual environments. For example, examining and refining the concepts proposed here by using Massive Open Online Courses (MOOC) platforms could help researchers to develop a more generalizable framework. This preliminary study also suggests that future research should carefully examine the role of goal-setting and self-regulation on learning platforms. Examining the experiential learning needs is another potentially rich avenue of study for future iterations of this inquiry. As such, future research should empirically examine how experiential needs may be orchestrated to achieve the most meaningful and engaging experience for users, leading to greater learning and growth. Further examination of this framework may also allow educators to better plan experiential learning processes in other educational contexts such as science, medicine, and engineering. 


\section{References}

[1] G. Secundo, G. Mele, P. Del Vecchio, G. Elia, A. Margherita, and V. Ndou, "Threat or opportunity? A case study of digital-enabled redesign of entrepreneurship education in the COVID-19 emergency," Technol. Forecast. Soc. Change, vol. 166, no. April 2020, p. 120565, 2021, doi: 10.1016/j.techfore.2020.120565.

[2] F. Von Briel et al., "Researching Digital Entrepreneurship: Current Issues and Suggestions for Future Directions," Commun. Assoc. Inf. Syst., vol. 48, no. 1, pp. 4-8, 2021.

[3] C. Leong, S. L. Pan, D. E. Leidner, and J. S. Huang, "Platform leadership: Managing boundaries for the network growth of digital platforms," J. Assoc. Inf. Syst., vol. 20, no. 10, pp. 1531-1565, 2019, doi: 10.17705/1jais.00577.

[4] O. Malgonde, H. Zhang, B. Padmanabhan, and M. Limayem, "Taming complexity in search matching: Two-sided recommende systems on digital platforms," MIS Q. Manag. Inf. Syst., vol. 44, no. 1, pp. 49-84, 2020, doi: 10.25300/MISQ/2020/14424.

[5] K. Abhari, D. Williams, P. Pawar, and K. Panjwani, "Smart Entrepreneurial Systems: An Application of Deep Reinforcement Learning in Improving Entrepreneurship Mentorship," in The Advances in Intelligent Systems and Computing, vol. 1364 Springer, 2021.

[6] S. Oviatt, A. Cohen, A. Miller, K. Hodge, and A. Mann, "The impact of interface affordances on human ideation, problem solving, and inferential reasoning," ACM Trans. Comput. Interact., vol. 19, no. 3, pp. 1-30, Oct. 2012, doi: $10.1145 / 2362364.2362370$.

[7] N. H. M. Rusok, N. Kumar, and S. M. A. Rahman, "A Contemporary Approach to Entrepreneurship Education and Training," Int. J. Asian Soc. Sci., vol. 7, no. 8, pp. 696-707, 2017.

[8] Y. C. J. Wu, T. Wu, and Y. Li, "Impact of using classroom response systems on students' entrepreneurship learning experience," Comput. Human Behav., vol. 92, pp. 634-645, 2019

[9] R. Harms, "Self-regulated learning, team learning and project performance in entrepreneurship education: Learning in a lean startup environment," Technological Forecasting and Social Change, vol. 100. pp. 21-28, 2015, doi: 10.1016/j.techfore.2015.02.007.

[10] A. Rauch and W. Hulsink, "Putting entrepreneurship Education where the intention to Act lies: An investigation into the impact of entrepreneurship education on entrepreneurial behavior," Acad Manag. Learn. Educ., vol. 14, no. 2, pp. 187-204, 2015, doi: 10.5465/amle.2012.0293.

[11] E. S. Mwasalwiba, "Entrepreneurship education: A review of its objectives, teaching methods, and impact indicators," Educ. Train., vol. 52, no. 1, pp. 20-47, 2010.

[12] R. Young, L. Wahlberg, E. Davis, and K. Abhari, "Towards a Theory of Digital Entrepreneurship Mindset: The Role of Digital Learning Aptitude and Digital Literacy," in AMCIS 2020 Proceedings, 2020, pp. 1-10.

[13] C. Bandera, R. Collins, and K. Passerini, "Risky business: Experiential learning, information and communications technology, and risk-taking attitudes in entrepreneurship education," Int. J. Manag. Educ., vol. 16, no. 2, pp. 224-238, 2018

[14] J. Morgan and D. Sisak, "Aspiring to succeed: A model of entrepreneurship and fear of failure," J. Bus. Ventur., vol. 31, no. 1, pp. 1-21, 2016, doi: 10.1016/j.jbusvent.2015.09.002

[15] R. K. Jena, "Measuring the impact of business management Student's attitude towards entrepreneurship education on entrepreneurial intention: A case study," Comput. Human Behav., vol. 107, no. December 2018, p. 106275, 2020

[16] H. Matlay, "The impact of entrepreneurship education on entrepreneurial outcomes," J. Small Bus. Enterp. Dev., vol. 15, no. 2, pp. 382-396, 2008, doi: 10.1108/14626000810871745.

[17] P. Rippa and G. Secundo, "Digital academic entrepreneurship: The potential of digital technologies on academic entrepreneurship," Technol. Forecast. Soc. Change, vol. 146, no. June 2018, pp. 900 911, 2019, doi: 10.1016/j.techfore.2018.07.013.

[18] P. Pintrich, "A conceptual framework for assessing motivation and SRL in college students," Educ. Psychol. Rev., vol. 16, no. 4, pp. 385-407, 2004.

[19] D. L. Butler, "Individualizing Instruction in," Spring, vol. 41, no. 2, pp. 81-92, 2002.
[20] E. Yukselturk and S. Bulut, "Predictors for student success in an online course," Educ. Technol. Soc., vol. 10, no. 2, pp. 71-83, 2007.

[21] Y. Zhu, W. Au, and G. Yates, "University students' self-control and self-regulated learning in a blended course," Internet High. Educ., vol. 30, pp. 54-62, 2016, doi: 10.1016/j.iheduc.2016.04.001.

[22] E. Ho, "Self-Regulated Learning and Academic Achievement of Hong Kong Secondary School Students.," Educ. J., vol. 32, no. 2, pp. 87-107, 2004.

[23] P. Bryant, "Self-regulation and moral awareness among entrepreneurs," J. Bus. Ventur., vol. 24, no. 5, pp. 505-518, 2009.

[24] L. W. Wardana et al., "The impact of entrepreneurship education and students' entrepreneurial mindset: the mediating role of attitude and self-efficacy," Heliyon, vol. 6, no. 9, 2020.

[25] X. Liu, C. Lin, G. Zhao, and D. Zhao, "Research on the effects of entrepreneurial education and entrepreneurial self-efficacy on college students' entrepreneurial intention," Front. Psychol., vol. 10, no. APR, pp. 1-9, 2019, doi: 10.3389/fpsyg.2019.00869.

[26] S. Vorbach, E. M. Poandl, and I. Korajman, "Digital Entrepreneurship Education: The Role of MOOCs.," Int. J. Eng. Pedagog., vol. 9, no. 3, 2019.

[27] D. Biduski, E. A. Bellei, J. P. M. Rodriguez, L. A. M. Zaina, and A. C. B. De Marchi, "Assessing long-term user experience on a mobile health application through an in-app embedded conversation-based questionnaire," Comput. Human Behav., vol. 104, no. October 2019, 2020, doi: 10.1016/j.chb.2019.106169.

[28] C. Lallemand, G. Gronier, and V. Koenig, "Computers in Human Behavior User experience: A concept without consensus? Exploring practitioners ' perspectives through an international survey," Comput. Human Behav., vol. 43, pp. 35-48, 2015.

[29] E. L. C. Law and X. Sun, "Evaluating user experience of adaptive digital educational games with Activity Theory," Int. J. Hum. Comput. Stud., vol. 70, no. 7, pp. 478-497, 2012, doi: 10.1016/j.ijhcs.2012.01.007.

[30] M. Adžgauskaitè, K. Abhari, and M. Pesavento, "How Virtual Reality Is Changing the Future of Learning in K-12 and Beyond," in HCI International 2020 - Late Breaking Papers: Cognition, Learning and Games. Lecture Notes in Computer Science, S. C, Ed. Cham: Springer, 2020, pp. 279-298.

[31] A. Sutcliffe and A. Alrayes, "Investigating user experience in Second Life for collaborative learning," Int. J. Hum. Comput. Stud., vol. 70, no. 7, pp. 508-525, 2012.

[32] K. Abhari, E. J. Davidson, and B. Xiao, "Inventing Together: The Role of Actor Goals and Platform Affordances in Open Innovation," J. Assoc. Inf. Syst., 2021.

[33] M. Uther and A. P. Banks, "The influence of affordances on user preferences for multimedia language learning applications," Behav. Inf. Technol., vol. 35, no. 4, pp. 277-289, 2016.

[34] R. Hartson, "Cognitive, Physical, Sensory, and Functional Affordances in Interaction Design," Behav. Inf. Technol., vol. 22, no. 5, pp. 315-338, Sep. 2003.

[35] S. Xue and D. Churchill, "A review of empirical studies of affordances and development of a framework for educational adoption of mobile social media," Educ. Technol. Res. Dev., vol. 67 , no. 5, pp. 1231-1257, 2019.

[36] E. Bonsignore, A. J. Quinn, A. Druin, and B. B. Bederson, "Sharing stories 'in the wild': A mobile storytelling case study using storykit," ACM Trans. Comput. Interact., vol. 20, no. 3, 2013, doi: 10.1145/2491500.2491506.

[37] D. Churchill and S. Xue, "Educational affordances of mobile social media for language teaching and learning: a chinese teacher's perspective," Comput. Assist. Lang. Learn., 2020.

[38] E. Karahanna, S. X. Xu, Y. Xu, and N. Zhang, "The needsaffordances-features perspective for the use of social media," MIS Q. Manag. Inf. Syst., vol. 42, no. 3, pp. 737-756, Sep. 2018.

[39] K. Abhari, E. Davidson, and B. S. Xiao, "Experience First”: Investigating Co-creation Experience in Social Product Development Networks," AIS Trans. Human-Computer Interact., vol. 11, no. 1, pp. 1-32, 2019.

[40] C. M. Gray, P. Parsons, A. L. Toombs, N. Rasche, and M. Vorvoreanu, "Designing an Aesthetic Learner Experience: UX, Instructional Design, and Design Pedagogy.," Int. J. Des. Learn., vol. 11 , no. 1 , pp. $41-58,2020$. 